\section{SOI: $1.1 /$ TAS $\quad$ DOI: $10.15863 /$ TAS \\ International Scientific Journal Theoretical \& Applied Science}

p-ISSN: 2308-4944 (print) e-ISSN: 2409-0085 (online)

Year: $2016 \quad$ Issue: 2 Volume: 34

Published: 29.02.2016 http://T-Science.org

\section{Nina Viktorovna Golubeva} candidate of technical Sciences, Associated Professor of the Department «Informatics, applied mathematics and mechanics», Omsk State Transport University, Russia znv.nvz@yandex.ru

SECTION 2. Applied mathematics. Mathematical modeling.

\title{
THE BASE INSTRUMENT OF SCIENTIFIC RESEARCH AND SOLVING ENGINEERING TASKS - MATHEMATICAL MODELING AT THE TECHNICAL UNIVERSITY
}

Abstract: The base scientific method of research and systems engineering of technical systems - mathematical modelling - plays an invaluable role in professional activity of the engineer and scientist. The most important component of process of formation of the future innovative engineer at technical University should be the mastering by tools, methods and approaches of mathematical modeling of systems and processes.

Key words: innovative engineer, mathematical modeling, the base scientific method, the mathematical apparatus, linear and nonlinear systems, static and dynamic models, the integral Laplace transformation, transfer function, state space.

Language: Russian

Citation: Golubeva NV (2016) THE BASE INSTRUMENT OF SCIENTIFIC RESEARCH AND SOLVING ENGINEERING TASKS - MATHEMATICAL MODELING AT THE TECHNICAL UNIVERSITY. ISJ Theoretical \& Applied Science, 02 (34): 152-156.

Soi: http://s-o-i.org/1.1/TAS-02-34-20 Doi: crossef http://dx.doi.org/10.15863/TAS.2016.02.34.20

\section{БАЗОВЫЙ ИНСТРУМЕНТ НАУЧНЫХ ИССЛЕДОВАНИЙ И РЕШЕНИЯ ИНЖЕНЕРНЫХ ЗАДАЧ - МАТЕМАТИЧЕСКОЕ МОДЕЛИРОВАНИЕ В ТЕХНИЧЕСКОМ УНИВЕРСИТЕТЕ}

Аннотация: Базовый научный метод исследования и проектирования технических систем математическое моделирование - играет неоченимую роль в профессиональной деятельности инженера и ученого. Важнейшей составляющей процесса формирования будущего инновационного инженера в техническом университете должно быть овладение инструментами, методами и подходами математического моделирования систем и процессов.

Ключевые слова: инновационный инженер, математическое моделирование, базовый научный метод, математический аппарат, линейные и нелинейные системы, статические и динамические модели, интегральное преобразование Лапласа, передаточная функияи, пространство состояний.

\begin{abstract}
Инженерно-технические и научные кадры являются главной движущей силой инновационного развития экономики, технологического перевооружения предприятий, создания новых наукоемких отраслей. Реалии жизни актуализируют необходимость обеспечить реальный сектор экономики специалистами качественно нового инновационного уровня. Качество инженерных кадров становится одним из ключевых факторов конкурентоспособности государства и, основой для его технологической, экономической независимости [1]. Наука и образование становятся базисными отраслями экономики [2].
\end{abstract}

Профессионализм, конкурентоспособность и востребованность инновационного инженера подразумевают владение комплексом ключевых компетенций, обладание глубокими фундаментальными и специальными знаниями, инновационным мышлением, способность интегрировать научное знание в инновационный продукт посредством передовых технологий, владение научными методами познания, исследования и проектирования.

Данная статья посвящена одному из важнейших компонентов инженерного образования - владению базовым научным методом исследования и проектирования технических систем - математическим 
моделированием. В процессе формирования инновационного инженера и в его будущей профессиональной деятельности эта универсальная научная методология играет неоценимую роль. Академик А. А. Самарский подчеркивал, что математическое моделирование является неизбежной составляющей научнотехнического прогресса [3].

Интенсификация развития техносферы порождает тенденции к усложнению инженерной деятельности, она приобретает все более интегрированный, комплексный и инновационный характер. Перед инженерами новой формации ставятся междисциплинарные, мультидисциплинарные, трансдисциплинарные задачи. Эти факторы обуславливают повышение значимости математического моделирования для успешной инновационной инженерной деятельности.

Авторский курс «Математическое моделирование систем и процессов» [4], который я читаю в Омском государственном университете путей сообщения (ОмГУПС), нацелен на раскрытие потенциала и возможностей математического моделирования для решения широкого спектра научных и инженерных задач.

Уделяется серьезное внимание принципам формирования математических моделей на основе различных категорий математического аппарата, методам решения и анализа моделей разных классов. Студенты знакомятся с классификациями математических моделей по форме представления, по характеру модели, по способу получения.

Одной из задач курса является формирование у студентов целостного представления о том, что характер создаваемой модели в каждом конкретном случае будет определяться поставленной задачей (целью исследования), объемом априорной информации о моделируемом объекте (процессе), режимом его работы, условиями, в которых он функционирует и другими факторами. Рассматриваются примеры построения и области применения различных по характеру математических моделей: статических и динамических, стационарных и нестационарных, линейных и нелинейных, непрерывных и дискретных, для систем с сосредоточенными и распределенными параметрами, детерминированных и стохастических [5].
Важно, чтобы у будущих инженеров в результате освоения данного курса выработалось четкое понимание следующих моментов.

Для исследования динамических свойств объекта формируется динамическая модель, в основу которой может быть положен математический аппарат дифференциальных уравнений, передаточных функций, пространства состояний, переходных функций, частотных передаточных функций и т. д. Категории математического аппарата для моделирования динамики технических систем представлены на рисунке 1. Выбор того или иного математического инструмента должен быть целесообразным и обоснованным.

Статические математические модели описывают установившиеся (равновесные) режимы работы физических систем [6]. В них фактор времени не учитывается. Для построения статических моделей используют математический аппарат систем линейных алгебраических уравнений, нелинейных алгебраических и трансцендентных уравнений, систем нелинейных алгебраических уравнений (рис. 1).

Различие методов и инструментов исследования и анализа линейных и нелинейных физических систем. Применимость принципа суперпозиции (наложения) существенно упрощает моделирование линейных систем. Рассматривая изучаемый объект как линейный, получают возможность применить для решения задачи мощный арсенал математических методов и инженерных инструментов исследования линейных систем, в том числе интегральное преобразование Лапласа, интеграл Дюамеля. Однако при этом всегда стоит помнить предостережение академика Н. Н. Моисеева о том, что «любые линейные модели не более чем аппроксимации реального нелинейного мира в определенной и весьма ограниченной области пространства и времени» [7].

При решении инженерных и научных задач часто возникает необходимость перехода от одной формы представления математической модели к другой (рис. 2), целью которого может быть достижение более четкого понимания различных аспектов поведения изучаемого объекта. Инженер должен владеть приемами и методами преобразования моделей [8]. 


\begin{tabular}{l|lrl|l|ll} 
& ISRA (India) & $=\mathbf{1 . 3 4 4}$ & SIS (USA) & $=\mathbf{0 . 9 1 2}$ & ICV (Poland) & $=\mathbf{6 . 6 3 0}$ \\
Impact Factor: & ISI (Dubai, UAE) $=\mathbf{0 . 8 2 9}$ & PUHU (Russia) $=\mathbf{0 . 1 7 9}$ & PIF (India) & $=\mathbf{1 . 9 4 0}$ \\
& GIF (Australia) & $\mathbf{0 . 5 6 4}$ & ESJI (KZ) & $=\mathbf{1 . 0 4 2}$ & & \\
& JIF & $=\mathbf{1 . 5 0 0}$ & SJIF (Morocco) $=\mathbf{2 . 0 3 1}$ & &
\end{tabular}



Рисунок 1 - Категории математического аппарата для построения статических и динамических моделей технических систем и процессов.

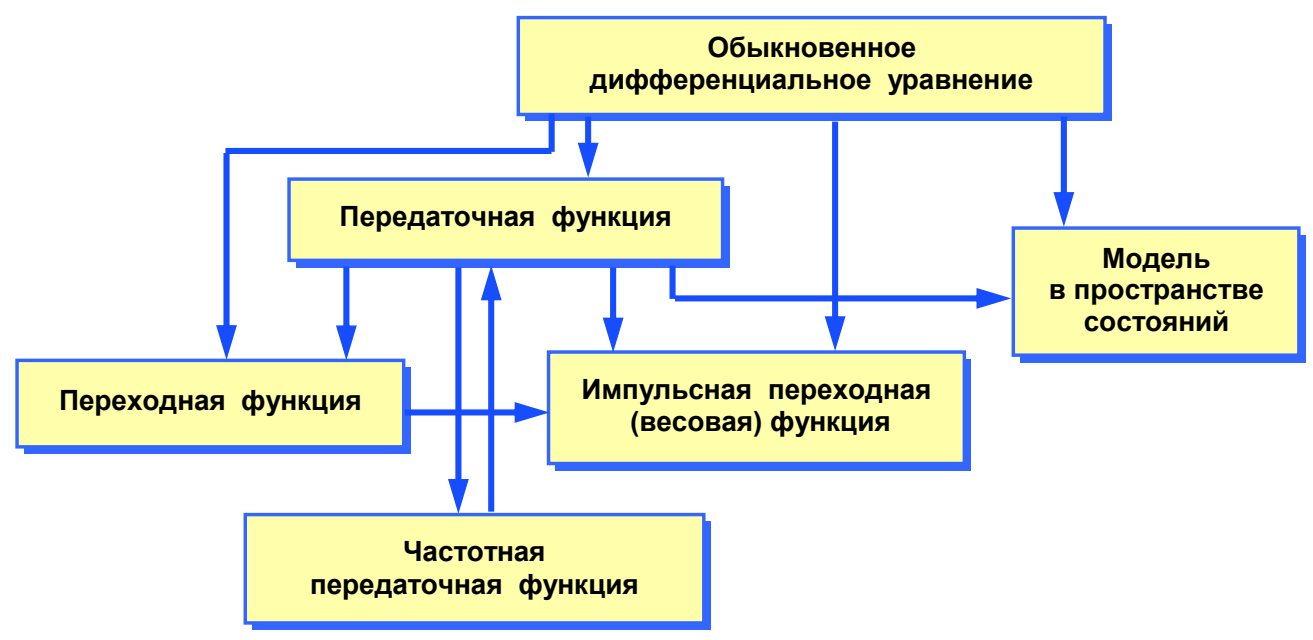

Рисунок 2 - Взаимосвязь форм представления математических моделей.

В курсе «Математическое моделирование систем и процессов» важное место отводится интегральному преобразованию Лапласа как эффективному инструменту решения широкого круга задач из различных технических областей. Рассматриваются примеры, демонстрирующие, что переход от исходных функций (оригиналов) к их изображениям значительно упрощает решение обыкновенных дифференциальных уравнений и расчет передаточных функций динамических систем [9]. Изучаются возможности 


\begin{tabular}{|c|c|c|c|c|c|c|}
\hline Impact Factor: & $\begin{array}{l}\text { ISRA (India) } \\
\text { ISI (Dubai, UAE } \\
\text { GIF (Australia) } \\
\text { JIF }\end{array}$ & $\begin{array}{l}=1.344 \\
=0.829 \\
=0.564 \\
=1.500\end{array}$ & $\begin{array}{l}\text { SIS (USA) } \\
\text { PИНЦ (Russia } \\
\text { ESJI (KZ) } \\
\text { SJIF (Moroccc }\end{array}$ & $\begin{array}{l}=0.912 \\
=0.179 \\
=1.042 \\
=2.031\end{array}$ & $\begin{array}{l}\text { ICV (Poland) } \\
\text { PIF (India) }\end{array}$ & $\begin{array}{l}=6.630 \\
=1.940\end{array}$ \\
\hline
\end{tabular}

математического аппарата передаточных функций.

Акцентируется внимание на существовании различных подходов к исследованию и анализу технических систем: во временной области и в частотной, детерминированный подход и стохастический. Раскрывается суть понятия «стохастическая модель». Формируются навыки оценивания допустимости или недопустимости применения детерминированной модели для описания исследуемого объекта при конкретной постановке задачи.

Рассматриваются принципы и примеры формирования теоретических моделей, в основу которых полагаются фундаментальные и феноменологические законы. Студенты знакомятся с методами и приемами построения эмпирических моделей, особенностями решения задачи аналитического приближения функций, полученных в результате эксперимента, с различными алгоритмами решения задач интерполяции.

Уделяется внимание вопросу качественного исследования динамических систем методом фазовой плоскости, позволяющего выявить общие свойства изучаемого объекта без явного интегрирования его дифференциального уравнения, что особенно важно при рассмотрении нелинейных технических систем.

Серьезное значение придается формированию у студента понимания сути, принципов, преимуществ и недостатков аналитических и численных методов решения.
Изучаются возможности различных современных интегрированных систем для реализации этих методов в контексте применения соответствующего математического аппарата.

Курс «Математическое моделирование систем и процессов» является междисциплинарным. В нем интегрируются научные знания, накопленный опыт, методы, приемы и концепции математики, физики, электротехники, информатики, кибернетики, теории электрических цепей, теории автоматического управления, электроники, теории систем и т. д. Успешное освоение этого курса, оптимально сочетающего в себе фундаментальные и прикладные аспекты, гарантирует формирование у студента столь востребованных сейчас междисциплинарных компетенций [10; 11].

Основная задача дисциплины «Математическое моделирование систем и процессов», по-мнению автора, научить будущего инженера применять накопленные им фундаментальные знания, приобретенные умения и навыки в своей профессиональной исследовательской и проектной деятельности, вооружить его инструментами, методами, принципами и алгоритмами трансформации этих знаний в инженерные решения прикладных областей.

\section{References:}

1. (2016) Oficial'nyi sait prezidenta Rossiiskoi Federacii / Stenogramma otchyota o zasedanii Soveta pri Prezidente po nauke i obrazovaniyu / Avaiable: http://www.kremlin.ru/transcripts/45962 (Accessed: 17.02.2016).

2. Gutenev VV (2016) Nauke prinadlezhit opredelyayushhaya rol $\mathrm{V}$ poluchenii reshayushhix konkurentnyx preimushhestv na osnovnyx napravleniyax razvitiya sovremennogo industrialnogo obshhestva / Avaiable:

http://www.soyuzmash.ru/news/vladimirgutenev-nauke-prinadlezhitopredelyayushchaya-rol-v-polucheniireshayushchih (Accessed: 17.02.2016).

3. Samarskij AA, Mixajlov AP (2002) Matematicheskoe modelirovanie: Idei. Metody. Primery. M.: Fizmatlit, 2002. - 320 p.
4. Golubeva NV (2013) Matematicheskoe modelirovanie sistem i processov: uchebnoe posobie. SPb.: Izdatelstvo «Lan», 2013. -192 p.

5. Golubeva NV (2015) Na puti k innovacionnomu inzhenernomu obrazovaniyu: maksimalnoe raskrytie vozmozhnostej nauchnogo metoda - matematicheskogo modelirovaniya. // Vestnik BGTU im. VG Shuxova. 2015. № 1. - pp. 220-222.

6. Tarasik VP (2004) Matematicheskoe modelirovanie texnicheskix sistem: uchebnik dlya vuzov. Mn.: DizajnPRO, 2004. - 640 p.

7. Moiseev NN (1997) Vremya opredelyat nacionalnye celi. M.: Izdatelstvo MNEPU, 1997. -256 p.

8. Dushin SE, Zotov NS, Imaev DX, Kuzmin NN, Yakovlev VB (2005) Teoriya avtomaticheskogo upravleniya: uchebnik dlya vuzov. 


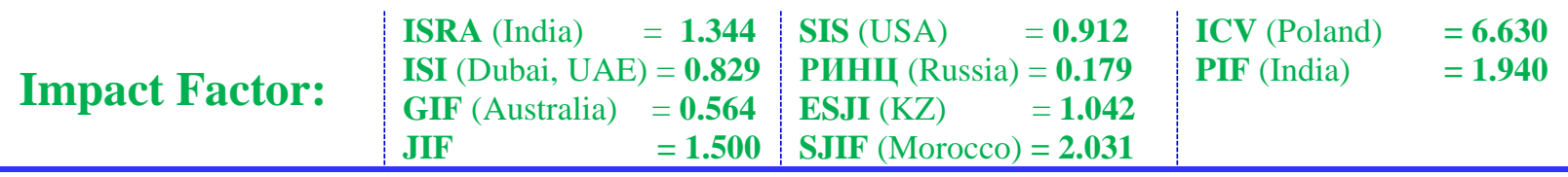

Pod red. V. B. Yakovleva. M.: Vysshaya shkola, 2005. - 567 p.

9. Besekerskij VA, Popov EI (2003) Teoriya sistem avtomaticheskogo upravleniya. SPb.: Izdatelstvo «Professiya», 2003. - 752 p.

10. Golubeva NV (2014) Matematicheskoe modelirovanie sistem i processov: ovladenie nauchnoj metodologiej budushhimi inzhenerami - put $\mathrm{k}$ professionalizmu. //
Theoretical \& Applied Science. 2014. № 1 (9). - pp.1-4.

11. Golubeva NV (2015) Matematicheskoe modelirovanie kak bazovyj instrument nauchnyx issledovanij: formirovanie inzhenera. // Sovremennaya nauka: aktualnye problemy i puti ix resheniya. 2015. № 6 (19). - pp. 12-14. 